\title{
Transition Probabilities in Argon I
}

\author{
C. H. Corliss and J. B. Shumaker, Jr. \\ Institute for Basic Standards, National Bureau of Standards, Washington, D.C. 20234
}

(April 27, 1967)

\begin{abstract}
In order to derive transition probabilities from intensity measurements of Ar I lines made by Dieke and Crosswhite, new transition probabilities for 26 lines from high levels in Ar I have been measured in a high current constricted arc. With these data, relative level populations of Ar I in Dieke and Crosswhite's micrewave discharge are determined and transition probabilities for 240 lines of Ar I in the wavelength range 4100 to $9800 \AA$ are derived. The new values are compared with other published values.
\end{abstract}

Key Words: Argon, atomic spectra, transition probabilities.

\section{Introduction}

In 1954 G. H. Dieke and H. M. Crosswhite published a report $[1]^{1}$ on the first spectrum of argon that included original intensity measurements made at The Johns Hopkins University spectroscopic laboratory. In the report, line intensities are tabulated for several types of discharges at argon pressures of 3 torr ${ }^{2}$ and below. In addition a photoelectric tracing of the argon spectrum produced by a microwave discharge in argon at 6.5 torr pressure is reproduced. The traces and the tabulated values are corrected for changes of response of the apparatus with wavelength by comparison with a calibrated tungsten lamp.

By using absolute transition probabilities for a number of lines whose upper energy levels are well distributed throughout the energy level structure of Ar I, we have been able to determine the relative populations of the energy levels excited in the argon microwave discharge and derive transition probabilities for several hundred lines measured in that discharge.

\section{Measurement of Lines from High Levels}

The relative populations of Ar I levels in the DiekeCrosswhite discharge were determined from $g A$ values measured in a consistent set of high-current constricted arc experiments [2,3]. Since these measurements included no lines with upper levels above $120000 \mathrm{~cm}^{-1}$, more arc measurements of lines from high levels were undertaken to permit calculation of Dieke-Crosswhite relative populations at higher energy levels. These measurements were made side-on

${ }^{1}$ Figures in brackets indicate the literature references at the end of this paper

${ }^{2}$ Editor's Note: 1 torr $=1 / 760$ standard atmospheres $=133.322 \mathrm{~N} / \mathrm{m}^{2}$. in a wall-stabilized arc operating in argon at 1 atm total pressure at currents of 30,40 , and $60 \mathrm{~A}$. In each experiment the continuum intensity at $4315 \AA$ was recorded during the usual side-on traverse of the arc. Then in a view only of the center, i.e., along an arc diameter, the spectral regions of interest were recorded. The intensity measurements were put on an absolute scale by recording the spectrum of a calibrated tungsten strip filament lamp placed in the arc position and with the arc window in the optical path. From an Abel inversion of the 4315 A continuum intensity the radial temperature distribution in the are was calculated using equilibrium argon composition tables [2] and the approximate continuum intensity expression [4]:

$I=1.63 \times 10^{-28} \gamma \xi(\lambda) N_{e}^{2} /\left(T^{1 / 2} \lambda^{2} Z_{+}\right)$ $\operatorname{erg} \mathrm{cm}^{-3} \mathrm{sr}^{-1} \mathrm{~s}^{-1} \AA^{-1}$

where $N_{e}$ is the electron number density, $T$ is the temperature, $Z_{+}$is the ion partition function, and $\gamma=6$ for argon. In a separate set of experiments carried out by methods previously described [2] a value for the Gaunt factor $\xi(4315 \AA)$ of 2.3 was obtained in good agreement with the value 2.4 reported by Morris et al. [5], for similar experiments. From the radial temperature distribution the number of excited atoms along an arc diameter was computed as a function of upper energy level again using the equilibrium composition tables and integrating across the source. The $g A$ values then follow directly by division of the measured line intensities by the number of emitters in the appropriate upper levels.

In order to minimize any systematic errors the 4300 $\AA$ Ar I transition probability was measured in each 
experiment along with the other lines and the scale factor required to produce $A_{4300}=4.11 \times 10^{5} \mathrm{~s}^{-1}$, the value reported in [2], was then applied to all the other $g A$ values. In every case the correction factor was within 11 percent of unity. The resulting arc measured transition probabilities are given in column 4 of table 1. Systematic errors arising, for example, from any inaccuracies involved in the integrations leading to the number of emitters along an arc diameter, or from the failure to record the spectrum exactly at the arc center, or from any uncertainty in $\xi(4315 \AA)$ are believed negligible. The fact that the transition probabilities proved to be independent of the arc current suggests that local thermodynamic equilibrium (L.T.E.) among the Ar I levels was maintained even at the low electron densities $\left(\sim 2 \times 10^{16}\right)$ of the $30 \mathrm{~A}$ arc, although this test is obscured in the high energy lines by the uncertainty in the large line wing corrections that were required.

Quantitative line wing corrections were made wherever practical by assuming dispersion profile line shapes [6] and, in addition, allowing for overlapping with other highly Stark broadened neighboring lines. In the comparatively favorable case of the narrow $4300 \AA$ line of Ar I which lies $30 \AA$ from its nearest neighbor this intensity correction amounts to 3 percent even in the $30 \mathrm{~A}$ arc. At the higher currents with $N_{e} \simeq 10^{17} \mathrm{~cm}^{-3}, 10$ to 15 percent of the line intensity lies beneath the apparent "continuum background" established by the intensities at 4285 and 4315 A. The transition probabilities obtained from the $60 \mathrm{~A}$ arc required such large wing corrections (factors of 2) for the lines from high levels that their accuracy is doubtful and we have omitted them from the averages presented in table 1 although their mean deviation from these averages is only 7 percent.

A few transitions are included in which no resolution of the arc experimental intensities into individual lines was possible. In these cases either the upper energy levels were sufficiently close together (e.g., 5738-40 $\AA, 4334-5 \AA$ ) that the total $g A$ value still has meaning, at least for most quasi-thermal sources, or, as in the case of the $6032 \AA$ Ar I line, a reasonably unambiguous resolution could be effected by the use of tentative relative $g A$ values from the Dieke and Crosswhite data. Since the Dieke-Crosswhite relative values in turn depended somewhat upon this resolution the procedure was necessarily an iterative one.

\section{Level Populations and Intensities in the Microwave Discharge}

With the aid of the transition probabilities of references [2] and [3] and the new measurements in table 1 we can examine the level populations in the discharges studied by Dieke and Crosswhite. We have chosen to work with the 6.5 torr microwave discharge for two reasons: the higher the pressure, the more nearly a discharge tends to exhibit an equilibrium population distribution and, furthermore, since the data are taken from tracings, it is possible to subtract the background radiation. It is evident by comparison with the tracings that their tabulated values have not been so corrected.

TABLE 1. New values and published values of transition probabilities for Ar I

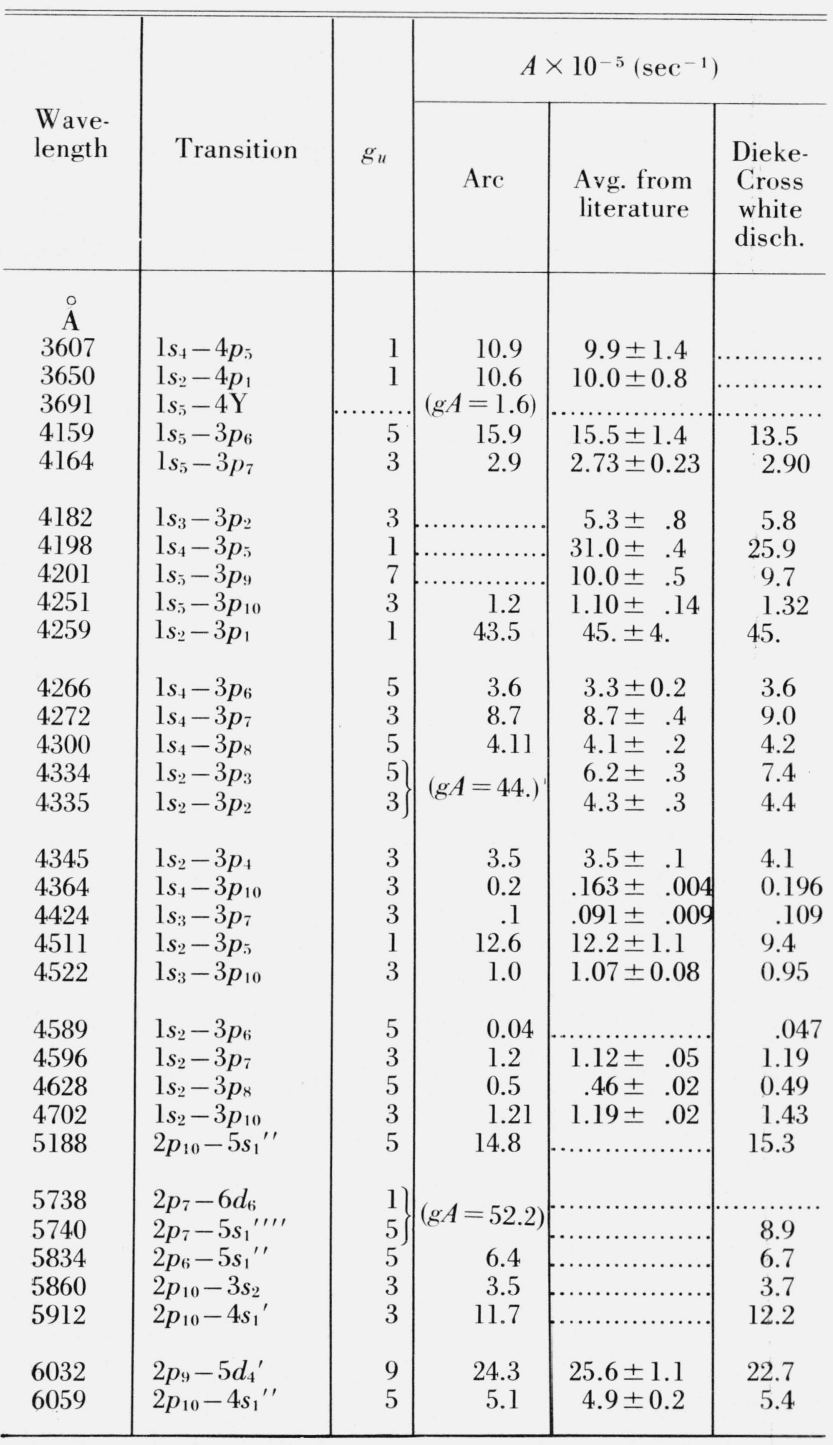

To determine the level populations from the data at hand, we note that the radiant power of a spectrum line emitted by $N_{u}$ atoms in an excited state $u$ is

$$
I=N_{u} h \nu A_{u l}
$$

where $A_{u l}$ is Einstein's transition probability or rate (per second) from an upper state $u$ to a lower state $l$. Since the number of magnetic sublevels in the upper state is $2 J_{u}+1=g_{u}$, the actual sublevel population is

$$
\frac{N_{u}}{g_{u}}=\frac{1}{h c} \cdot \frac{I \lambda}{g_{u} A_{u l}} .
$$




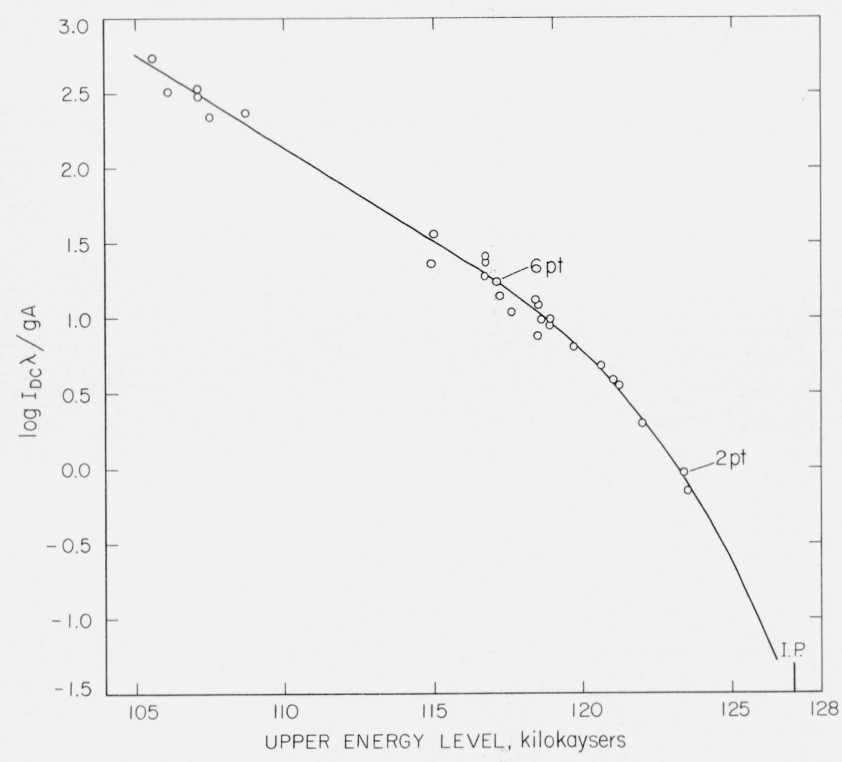

Figure 1. Population distribution among levels of Ar I in a 6.5 torr microwave discharge

In the expectation that the population of levels in the discharge will depend primarily upon the energy of the levels above the ground state we attempt to represent the population distribution among the levels of Ar I by plotting $\log I \lambda / g A$ as a function of $E$, the energy of the upper level. Such a plot for the 6.5 torr microwave discharge in argon is shown in figure 1 .

In a plot such as that in figure 1, a straight line would represent a thermal equilibrium population distribution. In this case the population of the lower levels is declining at a rate that would correspond to a temperature of $5000{ }^{\circ} \mathrm{K}$. Above about $117000 \mathrm{~cm}^{-1}$ the slope gradually grows steeper. The important aspect of the plot from our standpoint is that, within the range of scatter of the points, it defines a relationship between population and energy level value that is sufficiently regular to be used as an interpolation curve from which transition probabilities can be derived for the lines measured in the microwave discharge.

Before we can be sure that the intensity data are suitable for reduction to transition probabilities, we must find out if there is any self-absorption affecting the stronger lines and we must be sure that the intensity scale is correct as a function of wavelength. In order to detect any self-absorption in the intensity data, we calculated from the transition probabilities of Shumaker and Popenoe [3] unabsorbed relative intensities for the strong $4 s-4 p$ transitions of Ar I as they would appear in Dieke and Crosswhite's discharge. The appropriate upper level populations are taken from figure 1. A plot of the ratio of Dieke and Crosswhite's intensities to the calculated ones versus the calculated (unabsorbed) intensities in figure 2 shows that about three-quarters of the lines are self-

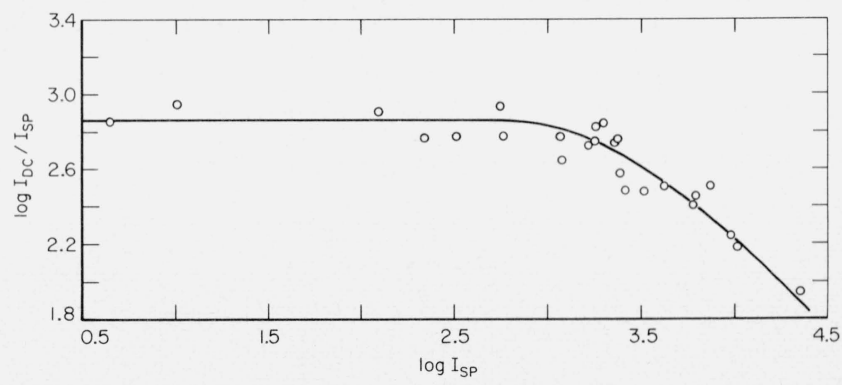

Figure 2. A plot of the ratio of Dieke and Crosswhite's intensities to unabsorbed intensities derived from Shumaker and Popenoe's transition probabilities versus the unabsorbed intensity.

All but seven of the lines in the $4 s-4 p$ transition array show some self-absorption.

absorbed in the microwave discharge. All values of $\log I_{\mathrm{DC}}$ larger than 5.7 are so affected. Only lines fainter than this have been plotted in figure 1.

All of the lines from upper levels higher than the $4 p$ levels are much fainter than the self-absorbed lines. The strongest of the high level lines is only 6 percent as intense as the weakest of the self absorbed lines. We have therefore assumed all of the lines from high levels to be free of self-absorption.

In discussing the accuracy of the intensity scale as a function of wavelength, we note, in the first place, that the scale of the tracings below $4000 \AA$ is ten times that of those above $4000 \AA$. Furthermore, a plot of the ratio of $A$-values calculated from Dieke and Crosswhite's intensities to those of Malone and Corcoran [7], who measured about $20 A$-values in this region, shows that Dieke and Crosswhite's intensity scale, after allowing for this scale shift, gradually declines below $4150 \AA$, until it is too small by about a factor of 10 at $3550 \AA$. For this reason we have not reported transition probabilities from their data below $4150 \AA$. Other comparisons (with data from table 1 and refs [2] and [3]) indicate that for the range 4150 to $8799 \AA$ their relative scale of intensities is correctly calibrated.

\section{Results From Dieke and Crosswhite's Measurements}

The results of our derivation of transition probabilities as outlined above are presented in tables 2 and 3 for 240 lines of Ar I. The wavelengths and transition designations (in Paschen's notation) are taken from Dieke and Crosswhite's report. The energy levels in kaysers $\left(\mathrm{cm}^{-1}\right)$ are taken from Moore's Atomic Energy Levels [8]. The statistical weights $(g=2 j+1)$ are listed next for the lower and upper levels respectively. The values of $\log I$ are read from the tracings in the D.-C. report and corrected for the background intensity level. The transition probabilities in the last column are given in units of $10^{5}$ per second. They are calculated with the formula

$$
A=\frac{\lambda}{g} \text { antilog }\left[\log I_{\mathrm{DC}}-\log \frac{I \lambda}{g A}\right]
$$




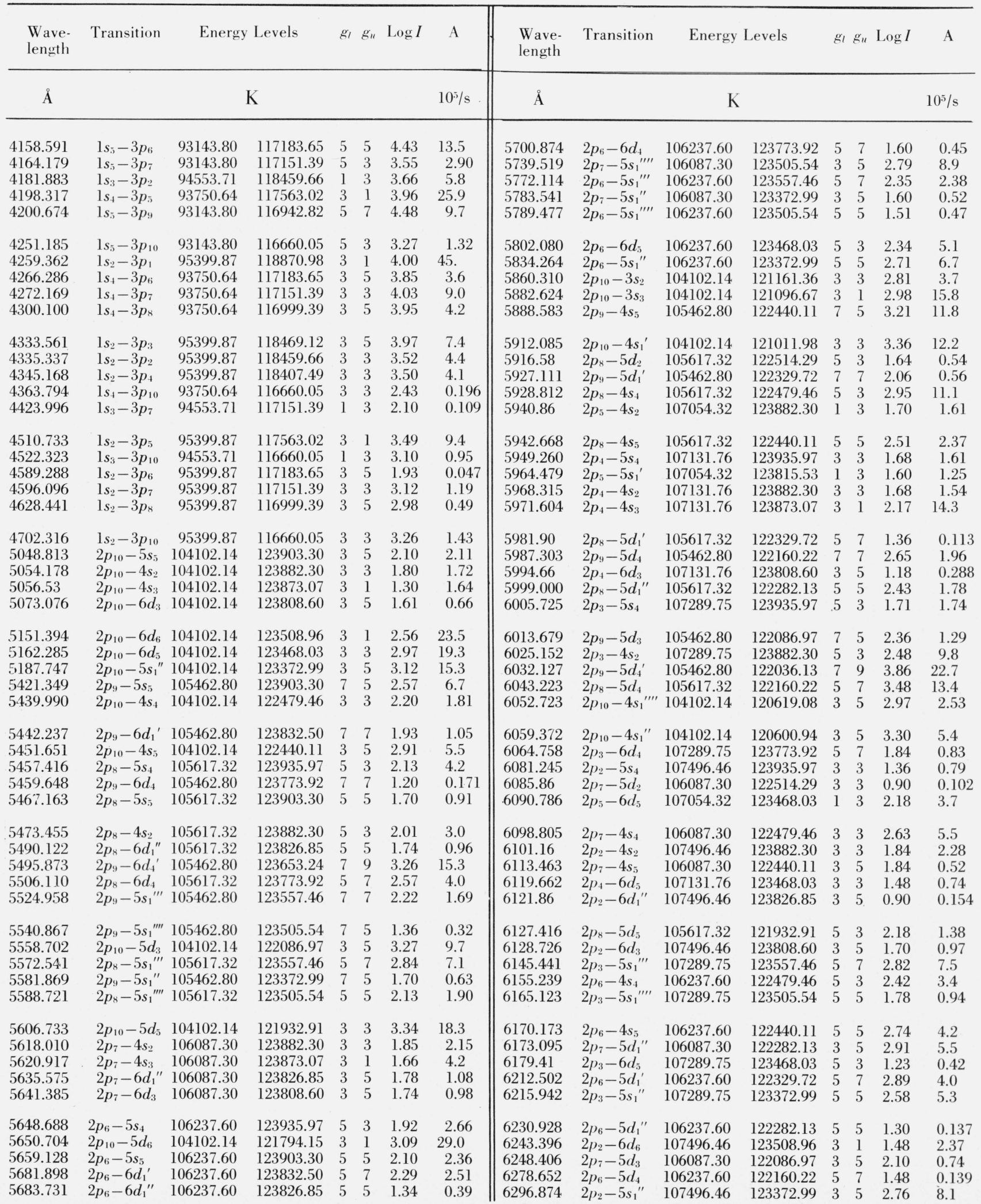


TABLE 2. Transition probabilities for lines of Ar I arising from levels below 124000 kaysers - Continued

\begin{tabular}{|c|c|c|c|c|c|c|c|c|c|c|c|c|c|c|c|}
\hline $\begin{array}{l}\text { Wave- } \\
\text { length }\end{array}$ & Transition & Energy & Levels & $g_{l}$ & $g_{u}$ & $\log I$ & A & $\begin{array}{l}\text { Wave- } \\
\text { length }\end{array}$ & Transition & \multicolumn{2}{|c|}{ Energy Levels } & $g_{l}$ & $g_{u}$ & $\log I$ & A \\
\hline$\AA$ & \multicolumn{6}{|c|}{$\mathrm{K}$} & $10^{5} / \mathrm{s}$ & $\AA$ & \multicolumn{6}{|c|}{ K } & $10^{5} / \mathrm{s}$ \\
\hline 6307.656 & $2 p_{6}-5 d_{3}$ & 106237.60 & 122086.97 & 5 & 5 & 3.00 & 5.9 & 7125.825 & $2 p_{4}-3 s_{2}$ & 107131.76 & 121161.36 & 3 & 3 & 3.06 & 8.0 \\
\hline 6309.14 & $2 p_{7}-5 d_{5}$ & 106087.30 & 121932.91 & 3 & 3 & 1.84 & 0.65 & 7147.041 & $1 s_{5}-2 p_{4}$ & 93143.80 & 107131.76 & 5 & 3 & 5.00 & 7.7 \\
\hline 6364.894 & $2 p_{7}-5 d_{6}$ & 106087.30 & 121794.15 & 3 & 1 & 2.23 & 4.5 & 7158.83 & $2 p_{4}-3 s_{3}$ & 107131.76 & 121096.67 & 3 & 1 & 3.11 & 26.0 \\
\hline 6369.576 & $2 p_{6}-5 d_{5}$ & 106237.60 & 121932.91 & 5 & 3 & 2.66 & 4.3 & 7162.57 & $2 p_{5}-4 s_{1}{ }^{\prime}$ & 107054.32 & 121011.98 & 1 & 3 & 2.11 & 0.83 \\
\hline 6384.716 & $2 p_{10}-3 s_{4}$ & 104102.14 & 119760.22 & 3 & 3 & 3.15 & 4.6 & 7206.981 & $2 p_{3}-3 s_{2}$ & 107289.75 & 121161.36 & 5 & 3 & 3.63 & 30. \\
\hline 6416.306 & $2 p_{10}-3 s_{5}$ & 104102.14 & 119683.11 & 3 & 5 & 3.83 & 12.8 & 7229.93 & $2 p_{8}-4 d_{1}^{\prime \prime}$ & 105617.32 & 119444.88 & 5 & 5 & 2.45 & 0.55 \\
\hline 6431.555 & $2 p_{8}-3 s_{2}$ & 105617.32 & 121161.36 & 5 & 3 & 2.08 & 0.76 & 7265.173 & $2 p_{7}-4 d_{2}$ & 106087.30 & 119847.81 & 3 & 3 & 2.88 & 2.91 \\
\hline 6466.550 & $2 p_{5}-5 d_{2}$ & 107054.32 & 122514.29 & 1 & 3 & 2.15 & 1.92 & 7270.66 & $2 p_{9}-4 d_{4}$ & 105462.80 & 119212.93 & 7 & 7 & 3.04 & 1.37 \\
\hline 6481.141 & $2 p_{5}-4 s_{4}$ & 107054.32 & 122479.46 & 1 & 3 & 1.48 & 0.41 & 7272.935 & $1 s_{4}-2 p_{2}$ & 93750.64 & 107496.46 & 3 & 3 & 5.29 & 17.2 \\
\hline 6493.971 & $2 p_{8}-4 s_{1}^{\prime}$ & 105617.32 & 121011.98 & 5 & 3 & 1.76 & 0.34 & 7285.44 & $2 p_{3}-4 s_{1}^{\prime}$ & 107289.75 & 121011.98 & 5 & 3 & 2.32 & 1.37 \\
\hline 6513.848 & $2 p_{4}-4 s_{4}$ & 107131.76 & 122479.46 & 3 & 3 & 1.65 & 0.61 & 7311.724 & $2 p_{7}-3 s_{4}$ & 106087.30 & 119760.22 & 3 & 3 & 3.49 & 11.4 \\
\hline 6538.112 & $2 p_{9}-4 s_{1}^{\prime \prime \prime}$ & 105462.80 & 120753.52 & 7 & 7 & 2.78 & 1.35 & 7316.007 & $2 p_{2}-3 s_{2}$ & 107496.46 & 121161.36 & 3 & 3 & 3.11 & 9.3 \\
\hline 6596.116 & $2 p_{9}-4 s_{1}^{\prime \prime \prime \prime}$ & 105462.80 & 120619.08 & 7 & 5 & 1.95 & 0.263 & 7350.78 & $2 p_{2}-3 s_{3}$ & 107496.46 & 121096.67 & 3 & 1 & 2.78 & 12.5 \\
\hline 6598.684 & $2 p_{3}-4 s_{5}$ & 107289.75 & 122440.11 & 5 & 5 & 1.70 & 0.41 & 7353.316 & $2 p_{8}-4 d_{4}$ & 105617.32 & 119212.93 & 5 & 7 & 4.00 & 12.6 \\
\hline 6604.854 & $2 p_{8}-4 s_{1}{ }^{\prime \prime \prime}$ & 105617.32 & 120753.52 & 5 & 7 & 3.07 & 2.66 & 7372.119 & $2 p_{9}-4 d_{4}^{\prime}$ & 105462.80 & 119023.70 & 7 & 9 & 4.45 & 25.9 \\
\hline 6632.087 & $2 p_{7}-3 s_{2}$ & 106087.30 & 121161.36 & 3 & 3 & 1.60 & 0.260 & 7392.97 & $2 p_{6}-3 s_{4}$ & 106237.60 & 119760.22 & 5 & 3 & 3.38 & 8.9 \\
\hline 6656.88 & $2 p_{2}-5 d_{2}$ & 107496.46 & 122514.29 & 3 & 3 & 1.40 & 0.35 & 7412.334 & $2 p_{4}-4 s_{1}^{\prime \prime \prime \prime}$ & 107131.76 & 120619.08 & 3 & 5 & 3.28 & 6.3 \\
\hline 6660.678 & $2 p_{7}-3 s_{3}$ & 106087.30 & 121096.67 & 3 & 1 & 2.60 & 7.5 & 7425.290 & $2 p_{3}-4 s_{1}^{\prime \prime \prime}$ & 107289.75 & 120753.52 & 5 & 7 & 3.23 & 4.3 \\
\hline 6664.053 & $2 p_{8}-4 s_{1}{ }^{\prime \prime \prime \prime}$ & 105617.32 & 120619.08 & 5 & 5 & 2.76 & 1.72 & 7435.33 & $2 p_{6}-3 s_{5}$ & 106237.60 & 119683.11 & 5 & 5 & 3.77 & 12.9 \\
\hline 6677.281 & $1 s_{4}-2 p_{1}$ & 93750.64 & 108722.67 & 3 & 1 & 3.96 & 3.1 & 7471.168 & $1 s_{4}-2 p_{4}$ & 93750.64 & 107131.76 & 3 & 3 & 3.51 & 0.261 \\
\hline 6684.73 & $2 p_{4}-5 d_{3}$ & 107131.76 & 122086.97 & 3 & 5 & 1.78 & 0.38 & 7484.24 & $2 p_{7}-4 d_{1}^{\prime \prime}$ & 106087.30 & 119444.88 & 3 & 5 & 3.43 & 5.4 \\
\hline 6698.875 & $2 p_{6}-3 s_{2}$ & 106237.60 & 121161.36 & 5 & 3 & 2.45 & 1.86 & 7510.42 & $2 p_{3}-4 s_{1}^{\prime \prime}$ & 107289.75 & 120600.94 & 5 & 5 & 3.18 & 5.1 \\
\hline 6719.219 & $2 p_{5}-5 d_{5}$ & 107054.32 & 121932.91 & 1 & 3 & 2.48 & 3.0 & 7618.33 & $2 p_{2}-4 s_{1}{ }^{\prime \prime \prime \prime}$ & 107496.46 & 120619.08 & 3 & 5 & 2.90 & 2.71 \\
\hline 6752.835 & $2 p_{10}-4 d_{3}$ & 104102.14 & 118906.66 & 3 & 5 & 4.28 & 28.2 & 7628.86 & $2 p_{2}-4 s_{1}^{\prime \prime}$ & 107496.46 & 120600.94 & 3 & 5 & 3.15 & 4.8 \\
\hline 6756.10 & $2 p_{3}-5 d_{3}$ & 107289.75 & 122086.97 & 5 & 5 & 2.68 & 3.0 & 7670.04 & $2 p_{8}-4 d_{5}$ & 105617.32 & 118651.45 & 5 & 3 & 2.90 & 1.98 \\
\hline 6766.613 & $2 p_{6}-4 s_{1}$ & 106237.60 & 121011.98 & 5 & 3 & 2.87 & 4.5 & 7891.078 & $2 p_{6}-4 d_{3}$ & 106237.60 & 118906.66 & 5 & 5 & 3.48 & 5.2 \\
\hline 6779.933 & $2 p_{1}-6 d_{5}$ & 108722.67 & 123468.03 & 1 & 3 & 1.70 & 1.36 & 8053.305 & $2 p_{6}-4 d_{5}$ & 106237.60 & 118651.45 & 5 & 3 & 3.26 & 4.8 \\
\hline 6818.291 & $2 p_{4}-5 d_{6}$ & 107131.76 & 121794.15 & 3 & 1 & 1.84 & 1.97 & 8605.779 & $2 p_{3}-4 d_{3}$ & 107289.75 & 118906.66 & 5 & 5 & 3.40 & 4.7 \\
\hline 6827.253 & $2 p_{3}-5 d_{5}$ & 107289.75 & 121932.91 & 5 & 3 & 2.34 & 2.22 & 8667.944 & $1 s_{3}-2 p_{7}$ & 94553.71 & 106087.30 & 1 & 3 & 5.54 & 24.0 \\
\hline 6851.884 & $2 p_{2}-5 d_{3}$ & 107496.46 & 122086.97 & 3 & 5 & 1.90 & 0.51 & 8761.691 & $2 p_{2}-4 d_{3}$ & 107496.46 & 118906.66 & 3 & 5 & 3.54 & 6.7 \\
\hline 6871.290 & $2 p_{10}-4 d_{5}$ & 104102.14 & 118651.45 & 3 & 3 & 4.23 & 38. & 9194.637 & $2 p_{10}-2 s_{2}$ & 104102.14 & 114975.07 & 3 & 3 & 4.42 & 24.9 \\
\hline 6879.59 & $2 p_{7}-4 s_{1}{ }^{\prime \prime \prime \prime}$ & 106087.30 & 120619.08 & 3 & 5 & 2.86 & 2.23 & 9291.58 & $2 p_{10}-2 s_{3}$ & 104102.14 & 114861.67 & 3 & 1 & 4.04 & 31. \\
\hline 6925.010 & $2 p_{2}-5 d_{5}$ & 107496.46 & 121932.91 & 3 & 3 & 2.28 & 1.96 & 9354.218 & $1 s_{2}-2 p_{7}$ & 95399.87 & 106087.30 & 3 & 3 & 5.11 & 9.6 \\
\hline 6937.666 & $2 p_{10}-4 d_{6}$ & 104102.14 & 118512.17 & 3 & 1 & 3.90 & 51. & 9784.501 & $1 s_{2}-2 p_{8}$ & 95399.87 & 105617.32 & 3 & 5 & 5.69 & 20.0 \\
\hline 6951.46 & $2 p_{6}-4 s_{1}{ }^{\prime \prime \prime \prime}$ & 106237.60 & 120619.08 & 5 & 5 & 3.06 & 3.6 & & & & & & & & \\
\hline 6960.23 & $2 p_{6}-4 s_{1}{ }^{\prime \prime}$ & 106237.60 & 120600.94 & 5 & 5 & 3.08 & 3.7 & & & & & & & & \\
\hline 6992.17 & $2 p_{2}-5 d_{6}$ & 107496.46 & 121794.15 & 3 & 1 & 2.58 & 11.1 & & & & & & & & \\
\hline 7030.252 & $2 p_{9}-3 s_{5}$ & 105462.80 & 119683.11 & 7 & 5 & 4.26 & 38. & & & & & & & & \\
\hline 7086.70 & $2 p_{5}-3 s_{2}$ & 107054.32 & 121161.36 & 1 & 3 & 2.40 & 1.75 & & & & & & & & \\
\hline 7107.478 & $2 p_{8}-3 s_{5}$ & 105617.32 & 119683.11 & 5 & 5 & 3.38 & 5.0 & & & & & & & & \\
\hline
\end{tabular}


TABLE 3. Transition probabilities for lines of Ar I with upper levels between 124500 and 126300 kaysers

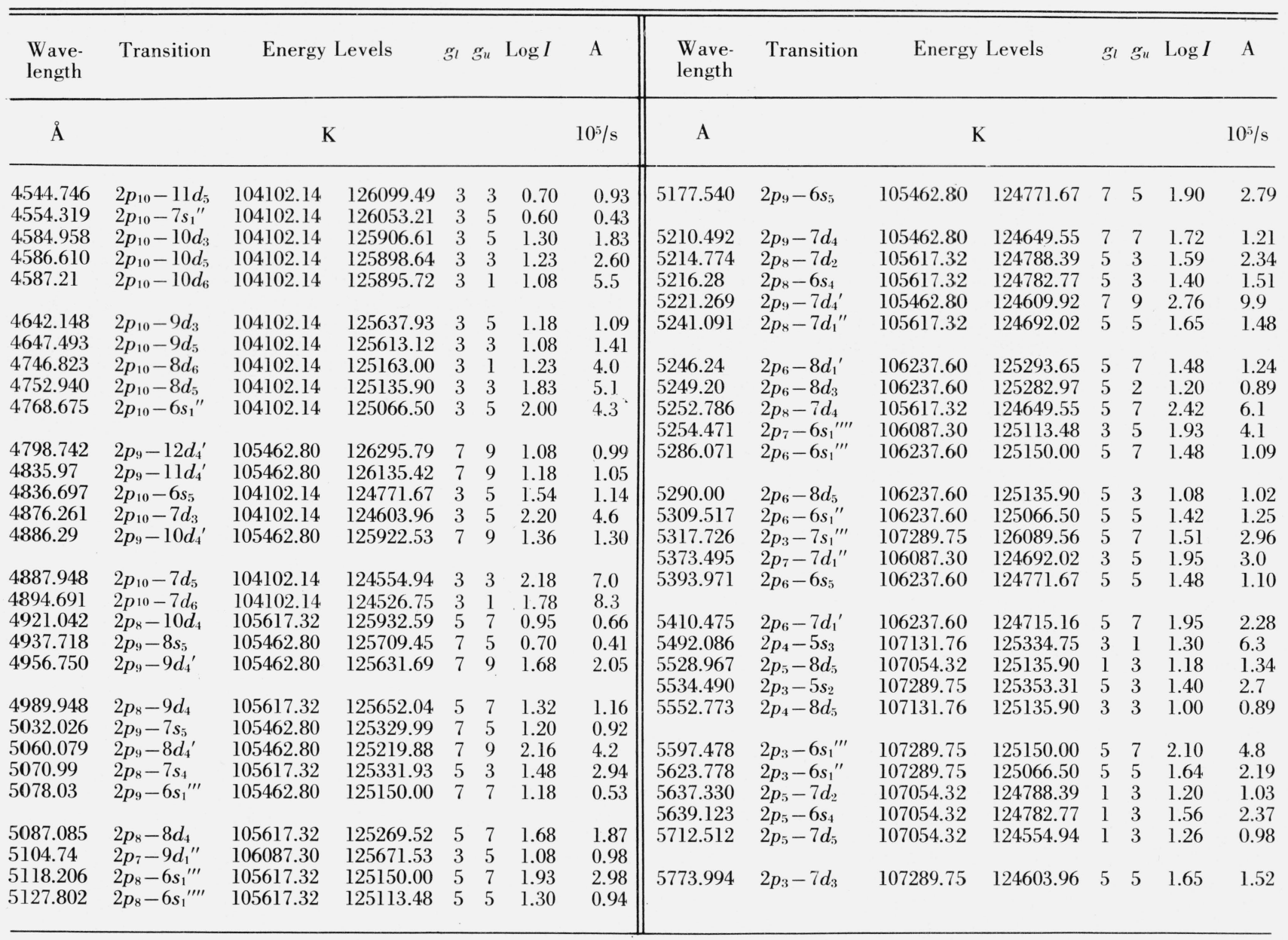

where $\lambda, g$, and $\log I_{\mathrm{DC}}$ are the values in tables 2 and 3 and $\log I \lambda / g A$ was read with a precision of $0.01(3 \%)$ from the ordinate of a large scale plot of figure 1 for each upper energy level.

The 184 lines reported in table 2 arise from levels in Ar I below $124000 \mathrm{~cm}^{-1}$ where the population curve in figure 1 is well calibrated. The 56 lines in table 3 arise from levels in the range of energy from 124000 to $126300 \mathrm{~cm}^{-1}$ where the population curve is extrapolated. If we assume that the error arising from the extrapolation does not exceed the difference between the curve as shown in figure 1 and a tangent line drawn to the curve at $124000 \mathrm{~cm}^{-1}$ then we can say that the uncertainty of the values in table 3 will gradually increase with increasing upper energy level to a maximum value of 60 percent at $126300 \mathrm{~cm}^{-1}$. Extrapolation is always an unsatisfactory procedure but the possibility of obtaining transition probabilities in this energy range is otherwise remote. In the highcurrent constricted arc experiments, for example, these energy levels are practically obliterated by level broadening and lowering of the ionization potential.

Some measure of the accuracy of the relative values of the Dieke-Crosswhite transition probabilities of table 2 can be obtained by comparison with mean relative values for those lines on which extensive reliable measurements have already been reported. For this purpose the arc transition probabilities reported above as well as those of Drawin [9], Gericke [10], Olsen [11], Popenoe and Shumaker [2], Bott [12], and Wiese [13] together with the shock tube values of Coates and Gaydon [14] and the RF heated plasma values of Malone and Corcoran [7] were averaged together as relative values and adjusted to the absolute transition probability scale used here. These mean values and the standard deviations of the individual determinations are shown in column 5 of table 1 . The corresponding Dieke-Crosswhite derived values from table 2 are shown in column 6 for comparison. The deviations of column 6 from the mean values in column 5 average 11 percent compared to an average standard deviation in column 5 of 7 percent.

The status of the absolute transition probabilities is somewhat less satisfactory. The absolute scale used throughout this paper is that which, in arc measurements, is consistent with electron densities determined from the shape of the $4861 \AA$ line of impurity hydrogen $[2,5,13,15]$. This scale is in agreement with the small 
number of direct lifetime measurements in Ar I [16] but leads to the conclusion that Ar II levels in the are experiments are not in L.T.E. [5]. However, if in such arc experiments complete L.T.E. is assumed and the plasma diagnostics are based upon the Fowler-Milne method [11] or Richter's generalization of it [4] instead of upon electron density measurements, then an absolute scale for Ar I transition probabilities generally about 25 percent lower is indicated. This latter interpretation of the experiments produces Ar I transition probabilities in agreement with shock tube measurements [14] and also Ar II transition probabilities consistent with Ar II lifetime measurements [17, 18]. At the present it is not clear which interpretation, if either, is correct and the possibility that our results are systematically too high must be admitted.

\section{References}

[1] G. H. Dieke and H. M. Crosswhite, The Spectrum of Ar I, The Johns Hopkins University, Department of Physics, Baltimore (1954).
[2] C. H. Popenoe and J. B. Shumaker, Jr., J. Res. NBS 69A (Phys. and Chem.) No. 6, 495 (1965).

[3] J. B. Shumaker, Jr. and C. H. Popenoe, J. Opt. Soc. Am. 57, 8 (1967).

[4] J. Richter, Z. Astrophysik 61, 57 (1965).

[5] J. C. Morris, R. L. Garrison, and R. U. Krey, private communication and Bull. Am. Phys. Soc. 12, 811 (1967).

[6] W. L. Wiese and J. B. Shumaker, Jr., J. Opt. Soc. Am. 51, 937 (1961).

[7] B. S. Malone and W. H. Corcoran, J. Quant. Spectrosc. Radiat. Transfer 6, 443 (1966).

[8] C. E. Moore, Atomic Energy Levels, NBS Circ. 467, I, 211 (1949).

[9] H.-W. Drawin, Z. Phys. 146, 295 (1956).

[10] W. E. Gericke, Z. Astrophysik 53, 68 (1961).

[11] H. N. Olsen, J. Quant. Spectrose. Radiat. Transfer 3, 59 (1963).

[12] J. F. Bott, Physics of Fluids 9, 1540 (1966).

[13] W. L. Wiese, Eighth Internat. Conf. Ion. Phenom. in Gases, Vienna (1967).

[14] P. B. Coates and A. G. Gaydon, Proc. Roy. Soc. (London) A293, 452 (1966)

[15] B. Wende, Z. Phys. 198, 1 (1967).

[16] J. Z. Klose, J. Opt. Soc. Am. 57, 1242 (1967)

[17] W. R. Bennett, Jr., P. J. Kindlmann, and G. N. Mercer, Appl. Opt. Suppl. 2, 34 (1965).

[18] J. Bakos, J. Szigeti, and L. Varga, Physics Letters 20, 503 (1966).

(Paper 71A6-473) 